\title{
Inter-terminal transfer between port terminals. A continuous mathematical programming model to optimize scheduling and deployment of transport units.
}

\author{
Pau Morales Fusco \\ Senior Researcher, CENIT - Centre d'Innovació del Transport \\ Dr. Giulia Pedrielli \\ National University of Singapore, Centre for Maritime Studies \\ Chenhao Zhou, A/Prof. Loo Hay Lee, A/Prof. Ek Peng Chew \\ National University of Singapore, Industrial \& Systems Engineering
}

\section{SUMMARY}

In most large port cities, the challenge of inter-terminal transfers (ITT) prevails due to the long distance between multiple terminals. The quantity of containers requiring movement between terminals as they connect from pre-carrier to on-carrier is increasing with the formation of the mega-alliances.

The paper proposes a continuous time mathematical programming model to optimize the deployment and schedule of trucks and barges to minimize the number of operating transporters, their makespan, costs and the distance travelled by the containers by choosing the right combination of transporters and container movements while fulfilling time window restrictions imposed on reception of the containers.

A multi-step routing problem is developed where transporters can travel from one terminal to another and/or load or unload containers from a specific batch at each step. The model proves successful in identifying the costless schedule and means of transportation. And a sensibility analysis over the parameters used is provided.

\section{INTRODUCTION}

In large port cities with multiple port terminals at a certain distance, the challenge of interterminal transfers (ITT) prevails due to the long distance between multiple terminals. This is the case, for instance, of:

- Busan, South Korea - New Port and City Port are separated by 40 kilometers through city traffic, which can cause a single direction trip to take 2-3 hours;

- Kaohsiung, China - Mainland is separated from the island terminals through a tunnel, a bottleneck, which again can cause a single container transfer to take 2-3 hours;

- Hong Kong, China - CT9 and the other Kwai Chung terminals are separated by 10 kilometers over public roads; 
- Rotterdam, the Netherlands - Maasvlakte 1 and 2 are quite close by sea, but the road distance between both yards takes up to 40 kilometers.

The quantity (or ratio) of containers requiring movement between terminals as they connect from pre-carrier to on-carrier is increasing with the formation of the megaalliances. Whereas 2, 3, 5 or 6 MLO's (mainline operators) share individual east-west deep-sea services, each potentially has its own feeder and intra-Asia (or intra-EU) network to connect to. This makes terminal discharge and load planning somewhat complex and fragmented, with a single mainline vessel maybe discharging cargo for 150 or more next vessel/voyage/discharge port combinations.

This phenomenon is further magnified in transshipment terminals like Singapore and Hong Kong. Due to the rapid container turnover and tight discharging/loading schedule, the ITT in transshipment terminals happens more frequently and requires to be handled as fast as possible which eventually leads to a high operation cost. Although terminals operators try to minimize such activities by optimizing vessel allocation, the ITT cannot be completely eliminated and still affects terminal performance significantly.

Additionally, Singapore faces the problem of a limited local trucking pool. The scheduling problem for ITT becomes the key to make truck runs to be round-tripped or triangulated, both from a cost and also capacity perspectives. Dwelling time for the cargo is also an issue to be minimized, or where certain time windows need to be met to ensure its transshipment at the destination terminal. Third party logistics providers (3PL) are responsible for the deployment of the ITT systems, which is the stated problem we wish to optimize.

This paper proposes a continuous time mathematical programming model to optimize the deployment and schedule of trucks or other transport units to minimize the number of operating transporters, their makespan, costs and the distance travelled by the containers by choosing the right combination of transporters and container movements while fulfilling time window restrictions imposed on reception of the containers, a NP-hard problem. The paper first introduces an overview on the existing literature on the interterminal transfer, afterwards the problem is described and some notations are provided. Following it, the mathematical model used is described and some early stage results are provided and discussed. The paper finishes discussing a possible heuristic to the problem and further applications of the model beyond ITT.

\section{LITERATURE REVIEW}

Inter terminal transportation is a problem timidly approached in the existing literature. Some research such the presented in Hendriks et al., (2012) or Lee et al., (2012) 
considered inter terminal transportation in the context of the berth allocation problems for vessels calling at multi-terminal ports such as Antwerp and Singapore, respectively. However, optimization of the inter terminal transportation is not the main goal of neither paper and cost and time between terminals were given as an input parameter and the effect of land transport congestion or transporter allocation are out of their scope.

ITT optimization research has mainly been produced at the planning stages of Rotterdam's Maasvlakte port. Starting with the studies by Ottjes and Duinkerken (1996) and up to Duinkerken and Günther (2007) and Schroër et al. (2014). In all this papers a model was proposed to simulate the entire ITT process and compare the effectiveness and efficiency of several transport systems such as trucks, barges, AGVs etc. The simulations increased complexity up to, Schroër et al. (2014), where traffic modeling was firstly introduced to the ITT in order to identify delays caused by congestion.

In parallel, Tierney et al. (2014) presented a novel integer programming model for the ITT also in Rotterdam's Maasvlakte. To minimize container delivery delay, the key components of ITT were taken into account, including traffic congestion, transporter types and processing times, and terminal configurations. A time-space network was used to model the transporter, where intersections are represented by nodes with a maximum capacity during each time period. In fact, each unit of time -a discrete variable in the model- is represented with a different set of nodes.

The methodology of Tierney et al. (2014) was taken one step further by Nieuwkoop et al. (2014), who also proposed a time-space network to model the transporter's movements within terminals but this time the costs of the vehicles and the containers delay were quantified and the goal was to determine the optimal vehicle configuration.

A different approach was presented by Mishra et al. (2013), to introduce stochasticity to the problem, in terms of uncertainty in the arrival, handling and traveling times of the containers. In this case a semi-opened queueing system was used. Handling was not dependent on the number of containers loaded. The paper focused on estimating the delays and makespan of all origin and destination pairs, and although successful, solved a different problem than the one presented here since all transporters returned (empty) to a central depot before being deployed again and were served in a first-in-first-out basis (no time window constraints considered).

In the case of Zhang et al. (2009), the problem is explained by means of a multi Traveling Salesman Problem with Time Windows and a reactive tabu search is proposed to solve it. However, the resulting problem is quite different from the one proposed here, since in the ITT problem all the cargo has to be loaded/discharged and therefore, the reposition of empty containers needs to be considered. 
Dumas et al. (1991), proposed a pickup and delivery problem with time windows (PDPTW) that closely resembles the problem formulated in this paper. In this case, $n$ requests were converted into a directed graph whose node set $G=(V, E)$ is divided into pickup nodes $P=(1,2, \ldots, n)$ and delivery nodes $D=(n+1, n+2, \ldots, 2 n)$. I.e. each request is specified by its pickup node and delivery node. The authors reached exact solutions for the minimization of the paths considered by solving a simple graph search using column generation. However, the complexity escalates fast.

Later on, Qiu and Feuerriegel (2014), proposed a methodology similar to Dumas et al. (1991) to solve the ITT problem, this time with a multi-traveling salesman problem with time windows approach with selective pickup and delivery and transporter routing. A simple graph search considering both capacity and time window constraints is used, the routing for each transporter is designed to maximize the profit and it strongly depends on the selected delivery requests and the double nodes for container is used as well.

\section{PROBLEM DESCRIPTION}

In a preliminary stage the problem was addressed with a similar approach to those of Dumas et al. (1991) or Qiu and Feuerriegel (2014), aiming to the efficiency of truck usage, thanks to an optimized scheduling of the resources. The problem with either approach is that complexity escalates fast with the number of units being transported, since there are $4 I^{2}+I$ connections (arcs) in total to be checked for just $I$ containers to transfer. Therefore, the approach finally taken is completely different.

\subsection{Assumptions and Notations}

In the following, we propose a mathematical programming model to optimize the schedule of the trucks given that the total number is given. The objective function is to minimize the makespan for each container that will be transported by truck and the size of the pool of trucks (or transporters) being used.

Each transporter is considered to perform a sequential series of actions combining loading and unloading of containers and travelling between terminals. Therefore, the timeline of a given transporter can be expressed by means of a succession of steps, as expressed in Fig 1, characterized by the main action taking place (picking up or delivering) and, when necessary, travelling. An action is defined per each container involved, therefore, successive loading or unloading actions take place in differentiate steps. 


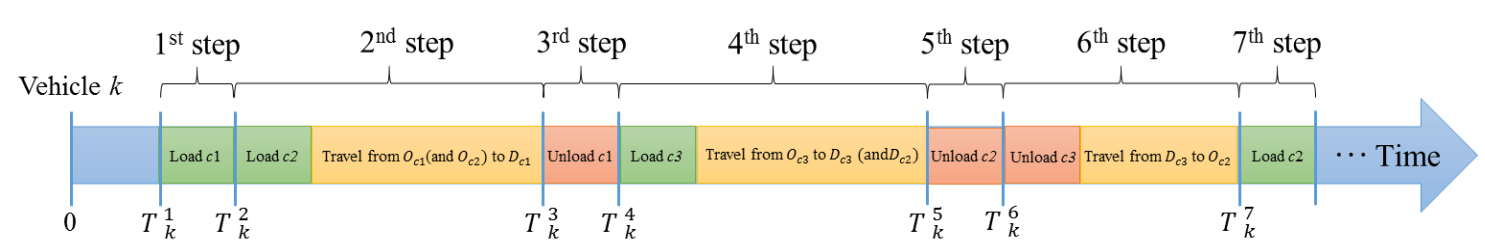

Fig 1. Successive actions with time steps approach

The goal will be building circular routes for each transporter that minimize the pool of transporters used and the makespan of their trips, while fulfilling certain time windows at the destination terminal (the container must be delivered during certain timeframe) and considering the availability at the origin.

Additionally, it is considered that transporters have capacity for more than one container (this would be the case of trucks transporting two TEU, twenty equivalent unit, containers for instance). This means that transporters will not necessarily travel directly from origin $i$ to destination $j$ of a given container $i$, since the transporter is allowed to visit different origins and pick up different containers (as far as its capacity is not surpassed) before delivering them. Therefore, size the container size is a value to be considered as well.

\subsection{Mathematical programming model components}

The objective is to minimize the sum of the costs related to the total makespan $\left(T_{k}^{T}\right)$ for each transporter $k$, the fixed costs of hiring new trucks and personnel plus on-board inventory costs as expressed in equation 1 :

$$
\min C^{T} \sum_{k=1}^{K}\left(T_{k}^{T}-T_{k}^{1}\right)+C^{F} \sum_{k=1}^{K}\left(1-q_{k}^{0}\right)+C^{I} \sum_{k=1}^{K} \sum_{t=1}^{T} \sum_{c=1}^{\mathbf{C}} y_{k c}^{t}
$$

Where $K$ is the set of all transporters considered, $T$ the number of time steps (actions performed by one transporter) and $\mathcal{C}$ the set of batches of containers considered. In turn, $C_{k}^{F}, C_{k}^{T}$ and $C_{k}^{I}$ are the fixed costs, costs per operator and operational costs for each transporter, respectively. $q_{k}^{0}$ is 1 for all transporters not being used and indicates that the transporter $k$ has finished all his activities at step $t$, and $y_{k c}^{t}$ is the amount of containers from the batch $c$ being at transporter $k$ at step $t$. To clarify concepts, operator cost represents the cost for operation time unit for transporter $k$. i.e., it is proportional to the transporter $k$ 's makespan $(\$ / h)$ while operational costs are costs per distance-weight unit for the transporter $k$ (\$/TEU-km), taking the weight of the average TEU, loaded, as weight unit.

Each batch of containers or commodity, $c$ belonging to the set $\mathcal{C}$ can be defined by $O_{c}$, terminal of origin; $D_{c}$, terminal of destination; $V_{c}$, volume or size of the batch; $S_{c}$, type (size) of the containers included in the batch $c$ ( 1 for 20 feet, 2 for 40 feet); $a_{c}$, time at which the batch will be available; and $e_{c}$ and $l_{c}$ vectors describing the daily earliest arrivals and the latest arrivals allowed for containers in the commodity. 
In turn each transporter (truck in this case) is defined by $C_{k}^{F}, C_{k}^{T}$ and $C_{k}^{I}$, as described before; $Q_{k}$, the capacity of transporter $k ; U_{k}, L_{k}$, the time to unload and load a container from the transporter $k$, respectivelly; and $T_{k i j}$, the travel time from terminal $i$ to terminal $j$ for transporter $k$.

In total, five decision variables are used to solve the problem:

- $\quad x_{k i c}^{\tau, t}$, amount of containers that are processed (i.e., loaded or unloaded) at terminal $i$ for the commodity $c$ during step $t$. The index $\tau=\{U, L\}$ refers to load or unload activity (only one of the two actions to be performed per step).

- $y_{k c}^{t}$, amount of commodity $c$ on transporter $k$ at step $t$, as introduced before.

- $v_{\text {kic }}^{t}, 1$ if the terminal $i$ is visited by transporter $k$ at step $t$. this serves the purpose to avoid that the transporter visits multiple ports during the same step.

- $T_{k}^{t}$, represents the time spent outside of the depot by truck $k$ at step $t$. From the very beginning to current step time.

- $q_{k}^{t}$, when 0 , indicates that the transporter is being used during step $t$. In fact it is used to identify whether a transporter is being used or not with $q_{k}^{t}=1$, or to indicate the last step with an action being performed by the actual transporter.

Equation 1 is subjected to the following constraints (equations 2-15)

$$
\begin{gathered}
\sum_{k=1}^{K} \sum_{t=1}^{T} x_{k i \mathrm{C}}^{L t}=V_{\mathrm{C}}, \quad \forall \mathrm{C} \in \mathbf{C}, i=O_{\mathrm{C}} \\
\sum_{k=1}^{K} \sum_{t=1}^{T} x_{k i \mathrm{C}}^{L t}=0, \quad \forall \mathrm{C} \in \mathbf{C}, i \neq O_{\mathrm{C}} \\
\sum_{k=1}^{K} \sum_{t=1}^{T} x_{k i \mathrm{C}}^{U t}=V_{\mathrm{C}}, \quad \forall \mathrm{C} \in \mathbf{C}, i=D_{\mathrm{C}} \\
\sum_{k=1}^{K} \sum_{t=1}^{T} x_{k i \mathrm{C}}^{U t}=0, \quad \forall \mathrm{C} \in \mathbf{C}, i \neq D_{\mathrm{C}} \\
\sum_{\tau=1}^{t} x_{k i \mathrm{C}}^{L \tau} \geq \sum_{\tau=1}^{t} x_{k j \mathrm{C}}^{U \tau}, \quad \forall k, \forall t, \forall \mathrm{C} \in \mathbf{C}, i=O_{\mathrm{C}}, j=D_{\mathrm{C}} \\
T_{k}^{t} \geq T_{k}^{t-1}+T_{k i j}^{t-1}-S_{\mathrm{C}} x_{k i \mathrm{C}}^{U t}+S_{\mathrm{C}} x_{k j \mathrm{C}}^{L t}, \quad \forall k, x_{k j \mathrm{C}}^{L t}+U_{k} \cdot x_{k j \mathrm{C}}^{U t}-\left(1-\sum_{\mathrm{C}} v_{k i \mathrm{C}}^{t-1}\right) \cdot M-\left(1-v_{k j \mathrm{C}}^{t}\right) \cdot M, j=O_{\mathrm{C}}, i=D_{\mathrm{C}}, t=1 \ldots T \\
T_{k}^{t} \geq T_{k}^{t-1}, \quad \forall k, t=2 \ldots T \\
T_{k}^{1} \geq T_{k}^{i n i t} \cdot\left(1-q_{k}^{0}\right), \quad \forall k \\
T_{k}^{t} \geq a_{\mathrm{C}} \cdot v_{k i \mathrm{C}}^{t}, \quad \forall k, t, \mathrm{C}, i=O_{\mathrm{C}}
\end{gathered}
$$




$$
\begin{gathered}
T_{k}^{t} \geq e_{\mathrm{C}} \cdot v_{k i \mathrm{C}}^{t}, \quad \forall k, t, \mathrm{C}, i=D_{\mathrm{C}} \\
T_{k}^{t} \leq l_{\mathrm{C}} \cdot v_{k i \mathrm{C}}^{t}+\left(1-v_{k i \mathrm{C}}^{t}\right) M, \quad \forall k, t, \mathrm{C}, i=D_{\mathrm{C}} \\
x_{k i \mathrm{C}}^{L t}+x_{k i \mathrm{C}}^{U t} \leq M \cdot v_{k i \mathrm{C}}^{t}, \quad \forall k, t, i, \mathrm{C} \\
x_{k i \mathrm{C}}^{L t}+x_{k i \mathrm{C}}^{U t} \geq v_{k i \mathrm{C}}^{t}, \quad \forall k, t, i, \mathrm{C} \\
\sum_{\mathrm{C}} \sum_{i \in\left\{O_{\mathrm{C}}, D_{\mathrm{C}}\right\}} v_{k i \mathrm{C}}^{t}=1-\sum_{\tau=1}^{t} q_{k}^{\tau}, \quad \forall t, k \\
\sum_{i \in I\left\{O_{\mathrm{C}}, D_{\mathrm{C}}\right\}} v_{k i \mathrm{C}}^{t}=0, \quad \forall t, k, \mathrm{C} \\
\sum_{\mathrm{C}} y_{k \mathrm{C}}^{t} \leq Q_{k}, \quad \forall k, t \\
y_{k \mathrm{C}}^{0}=0, \quad \forall k, \mathrm{C} \\
\sum_{t=0}^{T} q_{k}^{t}=1, \quad \forall k \\
q_{k}^{t-1}=\sum_{i \in\left\{O_{\mathrm{C}}, D_{\mathrm{C}}\right\}} \sum_{\mathrm{C}} v_{k i \mathrm{C}}^{t-1}-\sum_{i \in\left\{O_{\mathrm{C}}, D_{\mathrm{C}}\right\}} \sum_{\mathrm{C}} v_{k i \mathrm{C}}^{t} \quad \forall k, t=1,2 \ldots T
\end{gathered}
$$

Constraints (2) and (3) force the amount of loaded and the amount of unloaded containers from port $i$ to equal the demand for each commodity type. Note that the commodity really refers to the batch of containers having same origin and same destination.

Constraints (4) refer to the fact that it is not possible to unload a number of containers which is larger than the loaded ones. That is, establishes precedence.

Constraint number (5) models the inventory balance on board the truck at each step $t$, considering the size of the containers involved. In turn, constraints (6) control the activity time of each truck at step $t$, being $M$ a large enough number. Constraint (7) is dedicated to the first step for each transporter, establishing an starting time, subject to the time at which the transporter will be available, $T_{k}^{\text {init }}$.

Constraint (8) forces the pick up to take place after the container is available at origin while constraints (9) and (10) force the transporter activities to satisfy the earliest and latest times established at the final destination, that the time windows are met.

Constraints (11) link the binary variable $v_{k i c}^{t}$ and the integer variables $x_{\text {kic }}^{U, t}$ and $x_{\text {kic }}^{L, t}$, ensuring that a transporter can only perform a type of action per step. Constraints (12), in turn, force the transporter to perform only one activity during step $t$ as long as the $i$ port is either origin or destination of the commodity $c$ and the transporter is still on operation. 
Constraints (13) and (14) guarantee that the transporter capacity, the former establish the maximum number of containers at a given step while the later initialize the transporter's inventory.

Finally, constraints (15) identify the last step where transporter $k$ is performing an action and links variable $q_{k}^{t}$ with $v_{k i c}^{t}$.

\section{RESULTS AND DISCUSSION}

Several configurations varying the number of containers, vehicles, commodities, ports and maximum amount of time steps were considered to see the behavior of the experiment. The experiment was ran for 7200 seconds using CPLEX by IBM on a machine with an Intel Core 2 CPU 6300 at $1.86 \mathrm{GHz}$ with 4 GB of DDR2-533 RAM with the results described in Tables 1 to 4 :

\begin{tabular}{|c|c|c|c|c|c|c|c|c|}
\hline Port & Vehicle & Commodity & Container & Status & Time & $\begin{array}{c}\text { Obj } \\
\text { value }\end{array}$ & $\begin{array}{c}\text { Relative } \\
\text { Gap }\end{array}$ & Solutions \\
\hline 3 & 1 & 2 & 2 & Optimal & 0.3 & 524 & $0.0 \%$ & NA \\
\hline 3 & 1 & 4 & 4 & Optimal & 0.2 & 1727 & $0.0 \%$ & NA \\
\hline 3 & 1 & 6 & 6 & Optimal & 1.1 & 2031 & $0.0 \%$ & NA \\
\hline 3 & 1 & 8 & 8 & Optimal & 15.2 & 2535 & $0.0 \%$ & NA \\
\hline 3 & 1 & 10 & 10 & Optimal & 4.4 & 3698 & $0.0 \%$ & NA \\
\hline 3 & 2 & 2 & 4 & Optimal & 0.3 & 1148 & $0.0 \%$ & NA \\
\hline 3 & 2 & 4 & 8 & Optimal & 9.8 & 2904 & $0.0 \%$ & NA \\
\hline 3 & 2 & 6 & 12 & Feasible & 7200.0 & 2157 & $40.2 \%$ & 19 \\
\hline 3 & 2 & 8 & 16 & Feasible & 7200.2 & 4466 & $80.2 \%$ & 16 \\
\hline 3 & 2 & 10 & 20 & Feasible & 7200.0 & 5981 & $68.1 \%$ & 16 \\
\hline 3 & 3 & 2 & 6 & Optimal & 0.5 & 1672 & $0.0 \%$ & NA \\
\hline 3 & 3 & 4 & 12 & Optimal & 500.9 & 4632 & $0.0 \%$ & NA \\
\hline 3 & 3 & 6 & 18 & Feasible & 7200.0 & 6138 & $89.9 \%$ & 7 \\
\hline 3 & 3 & 8 & 24 & Feasible & 7200.0 & 7244 & $98.9 \%$ & 14 \\
\hline 3 & 3 & 10 & 30 & Feasible & 7200.0 & 9154 & $97.1 \%$ & 34 \\
\hline
\end{tabular}

Table 1. Results of the experiment ran varying commodities and vehicles

\begin{tabular}{|c|c|c|c|c|c|c|c|c|}
\hline Port & Vehicle & Commodity & Container & Status & Time & $\begin{array}{c}\text { Obj } \\
\text { value }\end{array}$ & $\begin{array}{c}\text { Relative } \\
\text { Gap }\end{array}$ & Solutions \\
\hline 3 & 3 & 2 & 6 & Optimal & 0.5 & 1672 & $0.0 \%$ & NA \\
\hline 3 & 3 & 4 & 12 & Optimal & 500.9 & 4632 & $0.0 \%$ & NA \\
\hline
\end{tabular}




\begin{tabular}{|c|c|c|c|c|c|c|c|c|}
\cline { 5 - 9 } 3 & 3 & 6 & 18 & Feasible & 7200.0 & 6138 & $89.9 \%$ & 7 \\
\hline 3 & 3 & 8 & 24 & Feasible & 7200.0 & 7244 & $98.9 \%$ & 14 \\
\hline 3 & 3 & 10 & 30 & Feasible & 7200.0 & 9154 & $97.1 \%$ & 34 \\
\hline 5 & 3 & 2 & 6 & Optimal & 0.4 & 3072 & $0.0 \%$ & NA \\
\hline 5 & 3 & 4 & 12 & Optimal & 424.0 & 5332 & $0.0 \%$ & NA \\
\hline 5 & 3 & 6 & 18 & Feasible & 7200.0 & 6738 & $95.0 \%$ & 19 \\
\hline 5 & 3 & 8 & 24 & No solution & 7200.0 & NA & $100.0 \%$ & 0 \\
\hline 5 & 3 & 10 & 30 & No solution & 7200.0 & NA & $100.0 \%$ & 0 \\
\hline
\end{tabular}

Table 2. Results of the experiment ran varying the number of ports

\begin{tabular}{|c|c|c|c|c|c|c|c|c|c|}
\hline $\begin{array}{l}\text { 는 } \\
\text { ○ }\end{array}$ & $\frac{\frac{0}{U}}{\frac{d}{d}}$ & 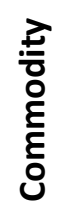 & 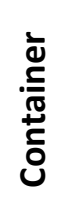 & $\stackrel{n}{\stackrel{n}{\Delta}}$ & $\underset{\stackrel{n}{D}}{\stackrel{n}{ \pm}}$ & $\stackrel{\mathscr{E}}{\underline{\boldsymbol{E}}}$ & $\begin{array}{l}\frac{0}{2} \\
\frac{0}{10} \\
\frac{0}{0}\end{array}$ & 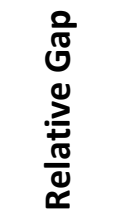 & 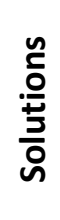 \\
\hline 3 & 2 & 10 & 20 & 20 & Feasible & 7200.0 & 5981 & $68.1 \%$ & 16 \\
\hline 3 & 2 & 10 & 20 & 25 & Feasible & 7200.0 & 5980 & $89.7 \%$ & 17 \\
\hline 3 & 2 & 10 & 20 & 30 & Feasible & 7200.0 & 7102 & $99.1 \%$ & 16 \\
\hline 3 & 2 & 10 & 20 & 35 & Feasible & 7200.0 & 5932 & $99.2 \%$ & 16 \\
\hline 3 & 2 & 10 & 20 & 40 & Feasible & 7200.0 & 5971 & $95.0 \%$ & 15 \\
\hline
\end{tabular}

Table 3. Results of the experiment ran varying the number of steps allowed

\begin{tabular}{|c|c|c|c|c|c|c|c|c|}
\hline Port & Vehicle & Commodity & Container & Status & Time & $\begin{array}{c}\text { Obj } \\
\text { value }\end{array}$ & $\begin{array}{c}\text { Relative } \\
\text { Gap }\end{array}$ & Solutions \\
\hline 3 & 2 & 4 & 4 & Optimal & 0.4 & 1447 & $0.0 \%$ & NA \\
\hline 3 & 2 & 4 & 8 & Optimal & 9.8 & 2904 & $0.0 \%$ & NA \\
\hline 3 & 2 & 4 & 12 & Optimal & 34.8 & 4912 & $0.0 \%$ & NA \\
\hline 3 & 2 & 4 & 16 & Optimal & 105.7 & 6820 & $0.0 \%$ & NA \\
\hline 3 & 2 & 4 & 20 & Optimal & 306.36 & 8676 & $0.0 \%$ & NA \\
\hline
\end{tabular}

Table 4. Results of the experiment ran varying the number of containers (commodities fixed)

As expected, the problem becomes more difficult to solve with the number of any of the parameters considered. It is to note that when allowing more steps (actions) per transporter or increasing the number of transporters, the complexity also escalates even if some of the transporters and steps, remain unused at the end, being extremely important to correctly size both terms from the beginning in order to reduce the time of computing.

In order to reduce complexity a multi-thread algorithm is being studied with the goal to find the right combination of vehicles and activities as described in Fig 2. The proposed methodology would start using a lower bound of steps and transporters to be considered until a feasible solution is found. From there, two different threads would be started, either increasing the number of steps or the number of vehicles to consider. 
In the first thread, the number of steps would be increased until there is no improvement on the solution found. In the second case -increasing the number of vehicles- the number of steps is to be reduced until the first infeasible case is found (there are not enough vehicles or steps). At this point a new thread would be opened by adding an extra vehicle. The final optimal solution would be found comparing the optimal solution found in each of the opened threads. Such approach is still under development.

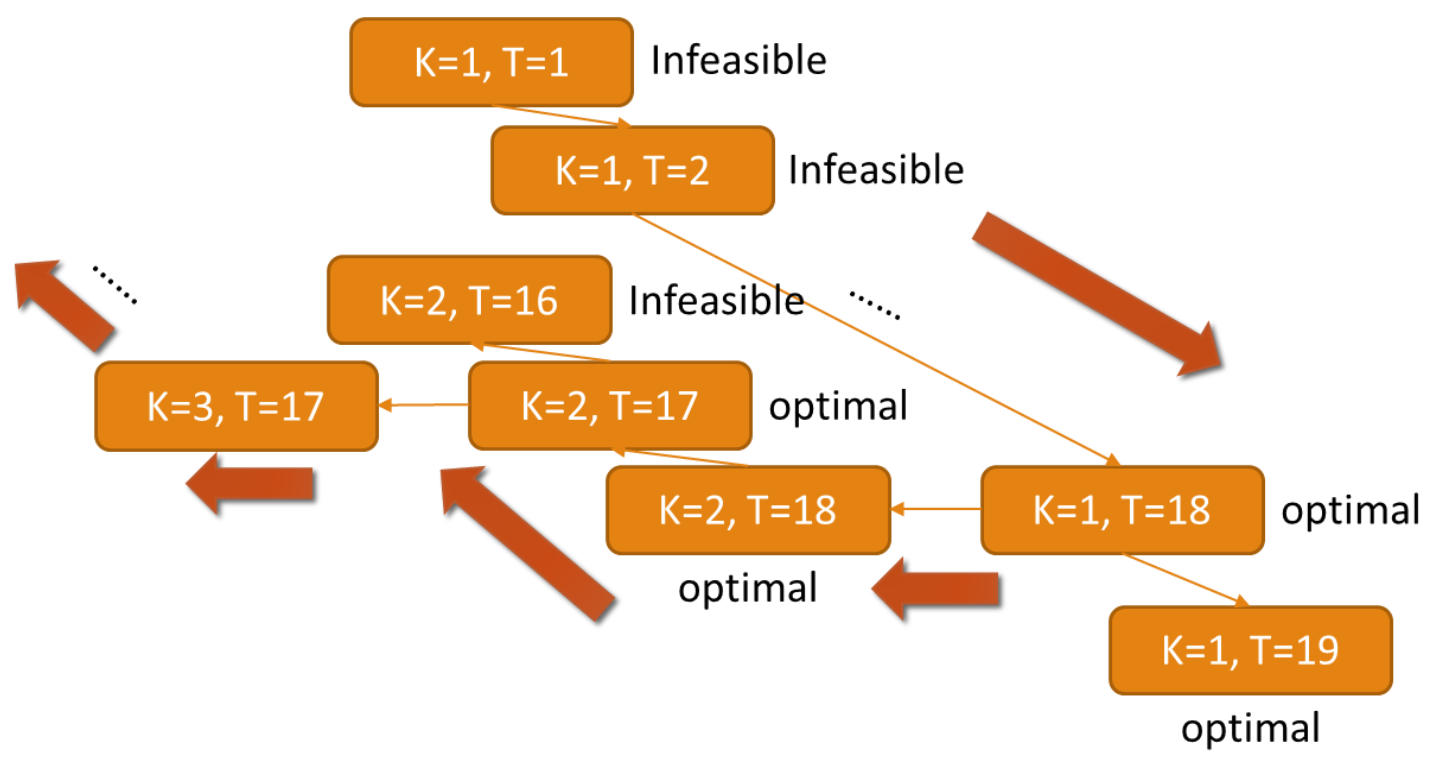

Fig 2. New proposed enumeration method

\section{CONCLUSIONS AND FURTHER RESEARCH}

This paper proposes a continuous time mathematical programming model to optimize the deployment and schedule of transporter units to minimize the number of operating transporters, their makespan, costs and the distance travelled by choosing the right combination of transporters and container movements while fulfilling time window restrictions imposed on reception of the containers.

A novel variation of the Pickup and Delivery Problem with Time Windows (PDPTW) is proposed to solve the problem after rejecting the use of a variation of the multi Traveling Salesman Problem introduced in Dumas et al. (1991) given the complexity of the graph obtained after small increases in the number of containers to be transferred.

The problem is approached as a sequential of activities or steps to be undertaken by each transporter unit successfully. However, computational times are still a burden and the right sizing of the pool of transporters and number of activities permitted to each of them must be solved to reduce the computing time. A multithread algorithm is sketch to find 
the right combination of transporters and steps to obtain the optimal solution but is not fully developed.

The methodology proposed, once finally solved, could also be applied to pools of transporters with varying characteristics. For instance, for the Inter Terminal Problem, consider barges as well as trucks to the transfer or trucks with different characteristics, such the megatrucks being implemented in Europe just recently. Additionally, it could be used to setup delivery schedules for express delivery problems in a urban context or the empty container reallocation problem for a global shipping liner.

\section{REFERENCES}

DUINKERKEN, M.B., DEKKER, R., KURSTJENS, S., OTTJES, J.A., DELLAERT, N.P., 2007. Comparing transportation systems for inter-terminal transport at the Maasvlakte container terminals, in: Kim, K.H., Günter, H.-O. (Eds.), Container Terminals and Cargo Systems. Springer Berlin Heidelberg, Berlin (Germany), pp. 3761.

DUMAS, Y., DESROSIERS, J., SOUMIS, F. (1991). The pickup and delivery problem with time windows. European Journal of Operational Research, 54, pp 7-22. HENDRIKS, M.P.M., ARMBRUSTER, D., LAUMANNS, M., LEFEBER, E., UDDING, J.T. (2012). Strategic allocation of cyclically calling vessels for multiterminal container operators. Flexible Services and Manufacturing Journal. 24, pp 248273.

LEE, D.-H., JIN, J.G., CHEN, J.H. (2012). Terminal and yard allocation problem for a container transshipment hub with multiple terminals. Transportation Research Part E Logistics and Transportiation Review. 48 (2), pp 516-528.

MISHRA, N., ROY D., OMMEREN, J.-K. (2013). A Stochastic model for interterminal container transportation. University of Twnete, Department of Applied Mathematics, the Netherlands.

NIEUWKOOP, F., CORMAN, F., NEGENBORN, R.R., DUINKERKEN, M.B., VAN SCHUYLENBURG, M., LODEWIJKS, G. (2014). Decision support for vehicle configuration determination in Inter Terminal Transport system design, in: Proceedings of the 11th IEEE International Conference on Networking, Sensing and Control, ICNSC 2014. pp. 613-618.

OTTJES, J.A., DUINKERKEN, M.B. (1996). Robotised inter-terminal transport of containers. A simulation study at the Rotterdam Port area, in: Proceedings of ESS 96. 8th European Simulation Symposium. Simulation in Industry. p. 621.

QIU, X., FEURRIEGEL, S. (2014). A multi-vehicle profit-maximizing pickup and delivery selection problem with time windows, in: European Conference on Information Systems (ECIS). AIS Electronic Library, Tel Aviv, Israel. SCHROËR, H.J.L., CORMAN, F., DUINKERKEN, M.B., NEGENBORN, R.R., LODEWIJKS, G. (2014). Evaluation of inter terminal transport configurations at Rotterdam Maasvlakte using discrete event simulation, in: Proceedings of the 2014 Winter Simulation Conference. IEEE Press, pp. 1771-1782.

TIERNEY, K., VOß, S., STAHLBOCK, R. (2014). A mathematical model of interterminal transportation. European Journal of Operational Research. 235, pp. 448-460. ZHANG, R., YUN, W.Y., MOON, I., 2009. A reactive tabu search algorithm for the multi-depot container truck transportation problem. Transportation Research Part E: 
Logistcs and Transportation Reviews, 45, pp 904-914. 